\title{
Article
}

\section{Trouble Articulating the Right Words: Evidence for a Response-Exclusion Account of Distraction During Semantic Fluency}

Marsh, John Everett, Crawford, Jessica Catherine, Pilgrim, Lea K., Sörqvist, Patrik and Hughes, Robert W.

Available at http://clok.uclan.ac.uk/18624/

Marsh, John Everett ORCID: 0000-0002-9494-1287, Crawford, Jessica Catherine, Pilgrim, Lea K. ORCID: 0000-0002-7739-0209, Sörqvist, Patrik and Hughes, Robert W. (2017) Trouble Articulating the Right Words: Evidence for a Response-Exclusion Account of Distraction During Semantic Fluency. Scandinavian Journal of Psychology . ISSN 0036-5564

It is advisable to refer to the publisher's version if you intend to cite from the work. http://dx.doi.org/10.1111/sjop.12386

For more information about UCLan's research in this area go to http://www.uclan.ac.uk/researchgroups/ and search for <name of research Group>.

For information about Research generally at UCLan please go to http://www.uclan.ac.uk/research/

All outputs in CLoK are protected by Intellectual Property Rights law, including Copyright law. Copyright, IPR and Moral Rights for the works on this site are retained by the individual authors and/or other copyright owners. Terms and conditions for use of this material are defined in the policies page. 


\begin{abstract}
It is widely held that single-word lexical access is a competitive process, a view based largely on the observation that naming a picture is slowed in the presence of a distractorword. However, problematic for this view is that a low-frequency distractor-word slows the naming of a picture more than does a high-frequency word. This supports an alternative, response-exclusion, account in which a distractor-word interferes because it must be excluded from an articulatory output buffer before the right word can be articulated (the picture name): A high, compared to low, frequency word accesses the buffer more quickly and, as such, can also be excluded more quickly. Here we studied the respective roles of competition and response-exclusion for the first time in the context of semantic verbal fluency, a setting requiring the accessing of, and production of, multiple words from long-term memory in response to a single semantic cue. We show that disruption to semantic fluency by a sequence of to-be-ignored spoken distractors is also greater when those distractors are low in frequency, thereby extending the explanatory compass of the response-exclusion account to a multiple-word production setting and casting further doubt on the lexical-selection-bycompetition view. The results can be understood as reflecting the contribution of speech output processes to semantic fluency.
\end{abstract}

Keywords: Semantic Verbal Fluency; Distraction; Distractor Frequency Effect; LexicalSelection-by-Competition, Response-Exclusion Account. 
The capacity for rapid, unimpeded, retrieval of words from the mental lexicon in long-term memory underpins many everyday activities such as the production of conversational speech and the naming of familiar objects. A central question within the literature on spoken word production concerns whether or not such lexical selection is a competitive process (Abdel Rahman \& Melinger, 2009; Finkbeiner \& Caramazza, 2006; La Heij, Kuipers, \& Starreveld, 2006; Mahon \& Caramazza, 2009; Mahon, Costa, Peterson, Vargas, \& Caramazza, 2007). The lexical-selection-by-competition account assumes that the latency to select a target representation for production is modulated by the activation levels of unselected non-target representations with highly activated non-target representations producing longer latencies. A major source of support for this view is the impact on single spoken-word production of distractor stimuli. For example, in the picture-word interference task, a distractor word (e.g., DOG) embedded in a picture (e.g., a house) slows the naming of that picture compared to a non-word (e.g., DGO) and especially if it is semantically related to the picture (e.g., DOG slows the naming of a picture of a cat more than does CHAIR). Classically, these lexical-semantic interference effects have been taken as strong evidence that lexical selection is a competitive process: as the lexical legality and semantic relatedness of the distractor word increases, so too does the competition between nodes within the mental lexicon. This competition slows selection of the target node (the picture name; e.g., Bloem \& La Heij, 2003; Levelt, 1999; Howard, Nickles, Coltheart, \& Cole-Virtue, 2006; Roelofs, 2003). An alternative account, however, supposes that the locus of such interference is at the later stage of articulatory output. According to this response-exclusion hypothesis (e.g., Mahon \& Caramazza, 2009), distractors block an articulatory output buffer at a post-lexical stage and picture naming is delayed due to the time it takes to exclude the distractor from the buffer. In the present study, we use distraction to study the possible roles of lexical competition and response-exclusion processes in the arguably more ecologically valid setting 
of multiple-word — as opposed to single-word—production: Our approach was to examine whether and how the vulnerability of producing a succession of category-exemplars from semantic memory (i.e., semantic verbal fluency; Bousfield \& Sedgewick, 1944; Newcombe, 1969) to distraction by spoken words is, like picture-word interference, modulated by the activation level of those words.

Typically, lexical-selection-by-competition and response-exclusion accounts have been contrasted in the context of the picture-word interference effect whereby a target picture (e.g., bed) is named more slowly when it is displayed concurrently with a to-be-ignored visual word (or a spoken word) as compared to a nonword (Glaser, 1992). In this setting, one of the strongest lines of evidence against the notion that lexical selection is a competitive process (e.g., Howard et al., 2006) is the effect of distractor-frequency. On the lexicalselection-by-competition model, the nodes representing high as compared with low frequency words should have a higher resting level of activation than low frequency words. Because of this, high frequency words should produce more interference (e.g., McClelland \& Rumelhart, 1981). High frequency distractors should activate competing nodes more than low frequency distractors, thereby slowing access to the target picture name (Finkbeiner \& Caramazza, 2006; Mahon \& Caramazza, 2009; Mahon, Costa, Peterson, Vargas, \& Caramazza, 2007; but see Roelofs, Piai, \& Schriefers, 2011). However, exactly the opposite pattern is found: Low frequency distractors impede picture-naming more than high-frequency distractors (Dhooge \& Hartsuiker, 2010; Miozzo \& Caramazza, 2003). Thus, a response-exclusion account that denies a role for competition at the lexical selection stage has been proposed (e.g., Miozzo \& Caramazza, 2003). In this account, distractor words gain access to an articulatory output buffer and have to be removed before a target word can be produced. Furthermore, the process of removing a distractor from the buffer depends on how fast the distractor occupies 
the output buffer. To the extent that low, compared to high, frequency words take longer to access the buffer, it also takes longer to exclude them (Miozzo \& Caramazza, 2003).

In the present article, we extend for the first time the investigation of the putative roles of lexical competition and response-exclusion to the multi-word production demands inherent in semantic fluency. Here, a semantic category-cue is presented (e.g., "vegetables”) and participants are required to produce as many exemplars from that category as they can in a given time-period. The semantic fluency task is thus assumed to tap fundamental processes involved in the sustained search for, and production, of context-appropriate lexical representations (Unsworth, Spillers, \& Brewer, 2011). Indeed, it is plausible that the difficulty of semantic fluency is determined to a large extent by competition at the lexicalselection stage from representations that are not context-appropriate given the presented cue. This is suggested by the selection-by-competition-account of lexical access (Perret, 1974; Rosen \& Engle, 1997). However, semantic fluency is also supported by speech output processes: in light of the semantic richness of the to-be-produced words coupled with the semantic criterion for word generation, the speech production system supports fluency by selecting the appropriate item from long-term memory for preparation for output (Knott, Patterson, \& Hodges, 2000; Martin \& Ayala, 2004; Wagner, Sebastian, Lieb, Tüscher, \& Tadić, 2014). Corroborating this, semantic fluency is impaired if covert articulation is prevented by articulatory suppression (Baddeley, Lewis, Eldridge, \& Thomson, 1984; Zhang \& Aitao, 2007). The effect of blocking the articulators on semantic fluency is characterised by a reduction in the size of subcategories produced (e.g., feline animals, beasts of burden) and the capacity to switch between them (Zhang \& Aitao, 2007). This suggests that covert articulation of items helps to cue the retrieval of others within the same subcategory or to keep track of items that have been produced to avoid repetition. A failure of the latter would lead to repeated sampling of prior responses (perserveration) thereby impairing fluency 
(Diaz, Sailor, Cheung, \& Kuslansky, 2004). Indeed, maintaining a concurrent 6-item memory load—which presumably involves some covert articulation or rehearsal of the memory load items - affects fluency by increasing repetitions of previous responses even if the load comprises items drawn from a category unrelated to the fluency category (Azuma, 2004; see also Rosen \& Engle, 1997).

A recent finding undergirding the present study is that semantic fluency is, like picture-naming, vulnerable to lexical distraction: to-be-ignored spoken words but not nonwords reduce the number of exemplars produced compared to a quiet control condition (Jones, Marsh, \& Hughes, 2012; Marsh \& Jones, 2010). From the standpoint that lexical selection is a competitive process, such vulnerability to spoken distractor-words during semantic fluency could be readily attributed to the distractors exacerbating the competition from inappropriate lexical representations at the word-selection stage. However, to the extent that speech production processes are also implicated in semantic fluency, an alternative possibility is that the distractors impede fluency by having to be excluded from an articulatory output buffer. We examine these possibilities in the present experiment by manipulating the frequency of the spoken distractors. Participants undertook semantic verbal fluency in the presence of a to-be-ignored sequence of low or high frequency words. Poorer fluency in the presence of high frequency words would suggest that lexical selection in a setting in which multiple words must be produced in succession is via competition (Roelofs, 2003). In contrast, poorer fluency in the presence of low frequency words would support a response-exclusion account which eschews the notion of lexical selection as a competitive process and emphasizes instead the late motoric-selection processes implicated when multiple events compete for action-control (Finkbeiner \& Caramazza, 2006; Mahon et al., 2007; Miozzo \& Caramazza, 2003). 


\section{Method}

\section{Participants}

Thirty-two undergraduate students at the University of Central Lancashire, all reporting normal or corrected-to-normal vision and normal hearing, participated in the experiment in return for course credit. All were native English speakers.

\section{Apparatus and Materials}

To-be-generated material. Thirty-two category-cues (e.g., "Four-legged Animals”) were selected from the Van Overschelde, Rawson, and Dunlosky (2004) norms. Categories were divided into two sets of 16 each. The potency of each cue (in terms of the mean number of responses produced to each cue) was matched across sets $(M=5.91, S E=.23 ; M=5.93$, $S E=.25 ; t(30)=-.073, p=.94)$.

Spoken distractors. The to-be-ignored spoken words comprised 11 high frequency words and 11 low frequency words taken from Miozzo and Caramazza (2003; see Appendix A). None of the to-be-ignored spoken words was semantically related to the visuallypresented category cue. Whilst the two sets differed significantly on lexical frequency, they were matched on other psycholinguistic dimensions (see Appendix B). Words were recorded in a male voice sampled with a 16-bit resolution at a rate of $44.1 \mathrm{kHz}$ using Audacity software (SourceForge). Each word was digitally edited to $500 \mathrm{~ms}$ using the sound compression function of Audacity. To produce a spoken sequence lasting $45 \mathrm{~s}$ (the length of time participants were given to produce exemplars in each semantic fluency trial; see below), 8 random permutations of the 11 words were presented back-to-back. The order of the permutations on a given trial was also random but care was taken to ensure that there were no immediate repeats of a given word. After exhausting the 8 random permutations, a further two words were chosen randomly (from the appropriate set) to achieve the desired duration of $45 \mathrm{~s}$. 


\section{Design}

A within-participant design was used with one factor, 'Frequency', incorporating two levels: high and low frequency distractors. The category-cues and irrelevant sounds were counterbalanced between participants such that each category-cue could appear equally often in the high and low distractor conditions. There were 32 trials, 16 for each distractor condition.

\section{Procedure}

Each participant was seated in an individual cubicle, seated at a viewing distance of approximately $60 \mathrm{~cm}$ from a PC monitor on which category-names were displayed in a central position. Category-names appeared in lower-case black 72-point Times New Roman font against a white background. Each category-name appeared for 45 s. Generation was immediate following the onset of the category-name.

Participants were informed that thirty-two category names would be presented one at a time on the computer monitor and that they were to generate as many words from each category as possible by speaking them into a microphone. One practice trial was presented (in quiet) before the experimental trials using a category-cue not used in the experimental trials. Participants were informed that they would have $45 \mathrm{~s}$ to generate as many words as they could per category-cue. Participants were instructed to ignore any sound heard through the headphones and were told that they would not be tested on its content at any point in the experiment.

\section{Results}

Fluency performance was assessed in terms of the total number of exemplars generated (discounting inappropriate responses and repeats). Fluency was poorer in the low frequency distractor condition $(M=10.1, S E=.78)$ than in the high frequency distractor condition $(M=10.8, S E=1.09)$. The difference between the two conditions was statistically 
significant, $t(31)=2.30, p=.029,95 \% C I[0.08,1.33]$, and had a medium effect size, $\mathbf{d}_{\mathrm{z}}=$ 0.41. It is worth noting that in terms of percentage difference, the size of the effect reported here—a 6.5\% difference between high and low frequency conditions-is larger than that reported in the picture-word interference paradigm (a difference of 2.5\%; 742 vs. 723 ms; Miozzo \& Caramazza, 2003; Experiment 1), as was the effect size (with $\mathbf{d}_{\mathrm{z}}=0.32$ in Experiment 1 of Miozzo \& Caramazza, 2003). Whilst caution must be exercised in comparing effect sizes with very different dependent variables (RT in picture-word interference vs. no. of exemplars generated in semantic fluency), the larger effect observed here may be related to the fact that, in the present setting, distraction was measured in terms of the impact of multiple distractors on multiple responses (see Discussion).

\section{Discussion}

The present study establishes a distractor-frequency effect in semantic verbal fluency. That low frequency distractors produce greater impairment in this setting than high frequency distractors is at odds with the lexical-selection-by-competition approach (e.g., Levelt, 1999) but is consistent with a response-exclusion account (e.g., Mahon et al., 2007) and extends the generality of the latter to multi-word production from semantic memory. One implication of the assertion that lexical selection is a non-competitive process, at least according to the response-exclusion account, is that the distractors are processed to a post-lexical level with the disruption arising at the stage of an output buffer. Within the context of the semantic verbal fluency used in the present study, this implies that the individual words within the tobe-ignored sequence of speech activate individual lexical entries that impede the production of to-be-generated items by occupying an output buffer (Miozzo \& Caramazza, 2003). This is broadly consistent with recent findings that demonstrate that neither the continuity of speech (i.e., the presence of co-articulatory cues) nor its inter-word semantic properties (i.e., semantic transitional probabilities) govern the disruption that it produces to semantically- 
based focal tasks (in such settings, to-be-ignored speech produces disruption due to its lexical- not supra-lexical level properties, see Marsh, Perham, Sörqvist, \& Jones, 2014).

An effect of distractor frequency has been found previously in the context of serial short-term memory (Buchner \& Erdfelder, 2005). In that study, low-frequency distractor words were more disruptive than high-frequency distractor words as they produced a larger disruption to recall of to-be-remembered sequences. One possible explanation for this particular finding is that low-frequency distractors may capture attention because their rarity or some other means by which they deviate from normality may signal importance. We argue here that the impairment of semantic verbal fluency by lexical distractors is not a consequence of attention capture but a consequence of the role of the speech production system in performing the focal task. We suggest that the delay in rejecting low frequency distractors from a response-output buffer-used in this setting to monitor previous responses and to cue yet to-be-recalled responses-impairs the production of a given target-exemplar which in turn impedes the fluency of the search-through-semantic-memory process. Whilst the relative timing between responses and the presentation of distractors cannot be controlled within the semantic fluency setting (unlike the picture-word interference paradigm), we speculate that, on average, the response buffer is likely to be occupied with a distractor when some of the exemplars are being prepared for output. That the relative timing between the onset of response and distractor presentation is perhaps less important than one might think in production-based tasks is supported by recent work within the picture-word interference paradigm. The distractor frequency effect persists even when the picture is presented some 1 $\mathrm{s}$ before the onset of the distractor (when participants are required to name the picture at the onset of the distractor; Dhooge \& Hartsuiker, 2011; Janssen, Schirm, Mahon, \& Caramazza, 2008). Such a finding contradicts the lexical-selection-by-competition approach which would 
predict no delayed interference effect from the distractor since lexical selection should have been completed before the distractor is presented (Janssen et al., 2008).

From a broader perspective, our results corroborate recent evidence of a close relation between language production and verbal memory wherein memory phenomena are interpreted in terms of constraints on the perceptual-motor systems involved in language processing (e.g., Acheson \& MacDonald, 2009; Hughes, Marsh, \& Jones, 2009; Perham, Marsh, \& Jones, 2009). By extension, the greater demand that the task places on the speechproduction system the more likely it is that distractor-variables-such as distractorfrequency — that impair speech-production will assume their disruptive potency. A recent finding that is broadly consistent with this view is that low output-dominant distractors (output-dominance being highly correlated with word frequency; Mervis, Catlin, \& Rosch, 1976) led to greater impairment in the context of a semantic category-clustering task than high dominant distractors when they are drawn from a category semantically unrelated to target categories (Marsh et al., 2014).

Whilst we have concentrated so far on the effects of distractor frequency, it is important to recognize that the semantic similarity between targets and distractors has a bearing on the patterns of disruption obtained within both semantic verbal fluency and picture-word interference tasks. For example, irrelevant speech comprising words drawn from a category semantically-related to the to-be-generated category (e.g., examples of "Fruit" when the target category was "Vegetables") produced greater disruption to fluency than categorically-unrelated words (e.g., "Carpenter's Tools"). This exacerbation of semantic distraction attributable to the semantic similarity between the to-be-generated material and irrelevant speech is also within the reach of the response-exclusion account. It has been assumed (Marsh, Beaman, Hughes, \& Jones, 2012; Marsh, Sörqvist, Hodgetts, Beaman, \& Jones, 2014) that the effect of between-sequence semantic similarity reflects the legacy of 
distractor inhibition whereby inhibitory activity spreads from distractor material to the semantically-related to-be-remembered items. This mechanism rests upon the assumption that distractors and targets compete to the extent that they share strong links with the parent category (Marsh et al., 2012). In the same way, a conflict could arise because to-be-ignored items, in the context of verbal fluency, activate potential responses that have to be excluded from the output buffer. Thus, the inhibition may not only act on the processing of background speech at the stage of encoding, but inhibition may also be responsible for the exclusion of erroneous responses at generation, in particular when these potentially erroneous responses are activated by the background speech.

Similarly, effects of semantic similarity between distractors and picture names can be explained by response exclusion and therefore without recourse to the notion of lexical competition (Mahon et al., 2007). In this setting, semantic interference effects have been found to be related to semantic distance (Mahon et al., 2007). For example, picture naming is slowed as distractor words become semantically-distant from the target picture. For example, the embedded distractor CRAB interferes with the naming of a picture of an "ant" compared to BEETLE, whilst BEETLE produces no interference compared to an unrelated distractor BOLT (the baseline condition). As Mahon and colleagues point out, the semantic distance effect is at odds with the lexical competition approach wherein the more semantically close the distractors are to the targets the greater the lexical competition and hence the slower the naming response should be. This is because the lexical nodes for closely related distractors and pictures should be more active and offer more competition due to the semantic priming between lexical entries. This suggests that the level of activation in non-target lexical nodes does not affect the time required to select target lexical nodes. According to the responseexclusion hypothesis, the semantic distance effect emerges because the process of removing a distractor from the articulatory output buffer uses semantic criteria. Therefore, words that are 
semantically-related to the to-be-named picture take longer to remove than unrelated distractors thereby giving rise to the semantic interference effect. However, the absence of a semantic interference effect from close category coordinates (e.g., "zebra" and HORSE) is due to those distractor words occupying the output buffer faster and therefore being excluded from the buffer faster (Mahon et al., 2007). Thus, an avenue for further research would be to examine whether such semantic-distance effects would also be evident in the context of semantic verbal fluency.

In sum, our finding that low frequency distractors are particularly disruptive of semantic verbal fluency, as is the case in single-word production (in the context of picture naming), suggests that the production of sequences of responses matching semantic criteria (semantic verbal fluency) is not underpinned by lexical-selection-by-competition. Further, we posit that semantic verbal fluency, like picture-naming, recruits speech production processes and that the impairment in semantic verbal fluency by distractors is attributable to the time taken to exclude them from response-output processes. Neither semantic verbal fluency under distraction nor picture-word interference, therefore, implicate competition at the lexicalselection level, only that contextually inappropriate words obligatorily occupy the articulatory output buffer thereby impeding fluent speech production. 


\section{References}

Abdel Rahman, A., \& Melinger, A. (2009). Semantic context effects in language production: A swinging lexical network proposal and a review. Language and Cognitive Processes, 24, 713-734.

Acheson, D. J., \& MacDonald, M. C. (2009). Twisting tongues and memories: Explorations of the relationship between language production and verbal working memory. Journal of Memory \& Language, 60, 329-350.

Azuma, T. (2004). Working memory and preservation in verbal fluency. Neuropsychology, 18, 69-77.

Baddeley, A. D., Lewis, V. J., Eldridge, M., \& Thomson, N. (1984). Attention and retrieval from long-term memory. Journal of Experimental Psychology: General, 113, 518-540.

Balota, D. A., Yap, M. J., Cortese, M. J., Hutchison, K. A., Kessler, B., Loftis, B., Neely, J. H., Nelson, D. L., Simpson, G. B., \& Treiman, R. (2007). The English lexicon project. Behavior Research Methods, 39, 445-459.

Bloem, I., \& La Heij, W. (2003). Semantic facilitation and semantic interference in word translation: Implications for models of lexical access in language production. Journal of Memory and Language, 48, 468-488.

Bousfield, W. A., \& Sedgewick, C. H. (1944). An analysis of sequences of restricted associative responses. Journal of General Psychology, 30, 149-165.

Brysbaert, M., Warriner, A. B., Kuperman, V. (2013). Concreteness ratings for 40 thousand generally known English word lemmas. Behavior Research Methods. DOI 10.3758/s13428-013-0403-5.

Buchner, A., \& Erdfelder, E. (2005). Word frequency of irrelevant speech distractors affects serial memory. Memory \& Cognition, 33, 86-97.

Dhooge, E., \& Hartsuiker, R. J. (2010). The distractor frequency effect in picture-word 
interference: Evidence for response exclusion. Journal of Experimental Psychology: Learning, Memory, \& Cognition, 36, 878-891.

Dhooge, E., \& Hartsuiker, R. J. (2011). The distractor frequency effect in a delayed pictureword interference task: Further evidence for a late locus of distractor exclusion. Psychonomic Bulletin \& Review, 18, 116-122.

Diaz, M., Sailor, K., Cheung, D., \& Kuslansky, G. (2004). Category size effects in semantic and letter fluency in Alzheimer’s patients. Brain \& Language, 89, 108-114.

Finkbeiner, M., \& Caramazza, A. (2006). Now you see it, now you don't: On turning semantic interference into facilitation in a Stroop-like task. Cortex, 42, 790-796.

Glaser, W. R. (1992). Picture naming. Cognition, 42, 61-106

Howard, D., Nickles, L., Coltheart, M., \& Cole-Virtue, J. (2006). Cumulative semantic inhibition in picture naming: Experimental and computational studies. Cognition, 100, 464-482.

Hughes, R. W., Marsh, J. E., \& Jones, D. M. (2009). Perceptual-gestural (mis)mapping in serial short-term memory: The impact of talker variability. Journal of Experimental Psychology: Learning, Memory, \& Cognition, 35, 1411-1425.

La Heij, W., Kuipers, J. R., \& Starreveld, P. A. (2006). In defense of the lexical-competition account of picture-word interference: A comment on Finkbeiner and Caramazza (2006). Cortex, 42, 1028-1031.

Janssen, N., Schirm, W., Mahon, B. Z., \& Caramazza, A. (2008). Semantic interference in a delayed naming task. Evidence for the response exclusion hypothesis. Journal of Experimental Psychology: Learning, Memory, \& Cognition, 34, 249-256.

Jones, D. M., Marsh, J. E., \& Hughes, R. W. (2012). Retrieval from memory: Vulnerable or inviolable? Journal of Experimental Psychology: Learning, Memory, \& Cognition, 38, 905-922. 
Knott, R., Patterson, K., \& Hodges, J. R. (2000). The role of speech production in auditoryverbal short-term memory: evidence from progressive fluent aphasia. Neuropsychologia, 38, 125-142.

Levelt, W. J. M. (1999). Models of word production. Trends in Cognitive Sciences, 3, 223232.

Mahon, B. Z., \& Caramazza, A. (2009). Why does lexical selection have to be so hard? Comment on Abdel Rahman and Melinger's swinging lexical network proposal. Language and Cognitive Processes, 24, 735-748.

Mahon, B. Z., Costa, A., Peterson, R., Vargas, K. A., \& Caramazza, A. (2007). Lexical selection is not by competition: A reinterpretive analysis of semantic interference and facilitation effects in the picture-word interference paradigm. Journal of Experimental Psychology: Learning, Memory, \& Cognition, 33, 503-533.

Marsh, J. E., Beaman, C. P., Hughes, R. W., \& Jones, D. M. (2012). Inhibitory control in memory: Evidence for negative priming in free recall. Journal of Experimental Psychology: Learning, Memory \& Cognition, 38, 1377-88.

Marsh, J. E., \& Jones, D. M. (2010). Cross-modal distraction by background speech: What role for meaning? Noise \& Health, 12, 210-6.

Marsh, J. E., Perham, N. R., Sörqvist, P., \& Jones, D. M. (2014). Boundary conditions for semantic auditory distraction: Lexicality and dominance act at retrieval. Memory \& Cognition, 42, 1285-1301.

Marsh, J. E., Sörqvist, P., Hodgetts, H. M., Beaman, C. P., \& Jones, D. M. (2014). Distraction control processes in free recall: Benefits and costs to performance. Journal of Experimental Psychology: Learning, Memory, \& Cognition, 41, 118-33.

Martin, N., \& Ayala, J. (2004). Measurements of auditory-verbal STM span in aphasia: Effects of item, task and lexical impairment. Brain \& Language, 89, 464-483. 
Mervis, C. B., Catlin, J., \& Rosch, E. (1976). Relationships among goodness-of-example, category norms, and word frequency. Bulletin of the Psychonomic Society, 7, 283284.

McClelland, J. L., \& Rumelhart, D. E. (1981). An interactive activation model of context effects. Psychological Review, 88, 375-407.

Miozzo, M., \& Caramazza, A. (2003). When more is less: A counterintuitive effect of distractor frequency in the picture-word interference paradigm. Journal of Experimental Psychology: General, 132, 228-252.

Newcombe, F. (1969). Missile wounds of the brain. Oxford University Press, London.

Perham, N., Marsh, J. E., \& Jones, D. M. (2009). Syntax and serial recall: How language supports short-term memory for order. Quarterly Journal of Experimental Psychology, 62, 1285-1293.

Perret, E. (1974). The left frontal lobe of man and the suppression of habitual responses in verbal categorization behavior. Neuropsycholgia, 12, 323-330.

Roelofs, A. (2003). Goal-referenced selection of verbal action: Modeling attentional control in the Stroop task. Psychological Review, 10, 88-125.

Roelofs, A., Piai, V., \& Schriefers, H. (2011). Selective attention and distractor frequency in naming performance: Comment on Dhooge and Hartsuiker (2010). Journal of Experimental Psychology: Learning, Memory, \& Cognition, 37, 1032-1038.

Rosen, V. M., \& Engle, R. W. (1997). The role of working memory capacity in retrieval. Journal of Experimental Psychology: General, 126, 211-227.

Unsworth, N., Spillers, G. J., \& Brewer, G. A. (2011). Variation in verbal fluency: A latent variable analysis of clustering, switching, and overall performance. Quarterly Journal of Experimental Psychology, 64, 447-466.

Van Overschelde, J. P., Rawson, K. A., Dunlosky, J. (2004). Category norms: An updated 
and expanded version of the Battig and Montague (1969) norms. Journal of Memory and Language, 50, 289-335.

Wagner, S., Sebastian, A., Lieb, K., Tüscher, O., \& Tadić, A. (2014). A coordinate-based ALE functional MRI meta-analysis of brain activation during fluency tasks in healthy control subjects. BMC Neuroscience, 15: 19.

Zhang, J., \& Aitao, L. (2007). The impact of the articulary loop and visuospatial sketchpad on phonemic and semantic fluency. Acta Psychologica Sinica, 39, 1012-1024. 
Appendix A

Distractor words used in the experiment.

\begin{tabular}{|c|c|}
\hline HF & LF \\
\hline bill & clump \\
\hline blood & diesel \\
\hline circle & farce \\
\hline fire & froth \\
\hline food & germ \\
\hline game & glove \\
\hline girl & hoop \\
\hline learn & prank \\
\hline list & pulse \\
\hline size & rib \\
\hline work & snag \\
\hline
\end{tabular}

Note: HF, high frequency; LF, low frequency. 


\section{Appendix B}

Psycholinguistic measures of distractors used in the experiment.

\begin{tabular}{|l|c|c|c|}
\hline Distractor Variable & HF & LF & Test Statistic \\
\hline K-F Frequency & $192.36(193.17)$ & $3.45(2.94)$ & $t(20)=3.24, p<.01$. \\
\hline Length (number of letters) & $4.36(.67)$ & $4.63(.81)$ & $t(20)=-.86, p>.05$. \\
\hline Number of syllables & $1.18(.4)$ & $1.09(.31)$ & $t(20)=.6, p>.05$. \\
\hline Word mean bigram frequency ${ }^{1}$ & 2756.81 & 2624.82 & $t(20)=-.36, p>.05$. \\
& $(1483.22)$ & $(1513.93)$ & \\
\hline Concreteness $^{2}$ & $4.19(.86)$ & $3.96(.81)$ & $t(20)=.64, p>.05$. \\
\hline
\end{tabular}

Note: 1 = measure obtained from Balota et al., 2007; 2 = measure obtained from Brysbaert, Warriner, and Kuperman, 2013. 